Schmerz 2011 25:365-367

DOI 10.1007/s00482-011-1075-7

(c) Deutsche Gesellschaft zum Studium des Schmerzes. Published by Springer-Verlag all rights reserved 2011

\author{
F. Petzke ${ }^{1} \cdot$ M. Pfingsten ${ }^{1} \cdot$ H.-R. Casser ${ }^{2} \cdot$ T. Tölle ${ }^{3} \cdot$ W. Koppert ${ }^{4}$ \\ ${ }^{1}$ Schmerzklinik im Zentrum Anästhesiologie, Rettungs- und Intensivmedizin, \\ Universitätsmedizin Göttingen \\ 2 DRK Schmerz-Zentrum Mainz \\ ${ }^{3}$ Klinik für Neurologie, TU München \\ ${ }^{4}$ Klinik für Anästhesiologie u. Intensivmedizin, Medizinische Hochschule Hannover
}

\title{
Ohne Struktur keine Qualität!
}

Die Versorgung von SchmerzpatienAufgrund unzureichender schmerztherapeutischer Versorgungsstrukturen beauftragte das Präsidium der Deutschen Gesellschaft zum Studium des Schmerzes (DGSS) Anfang 2010 eine Ad-hoc-Kommission mit der Aufgabe, Empfehlungen zur Strukturqualität in der Schmerzmedizin - ausgehend von den durch die International Association for the Study of Pain (IASP) bereits 1991 international geforderten Konzepten [1] und angepasst an die Situation in Deutschland - zu entwickeln. Die eingesetzte Kommission setzte sich aus Vertretern verschiedener Berufsgruppen und Versorgungsstrukturen zusammen. Die Kommission hat sehr schnelle und konstruktive Arbeit geleistet und legt in dieser Ausgabe von Der Schmerz ihre konsensbasierten Empfehlungen vor [2]. Das Präsidium der DGSS hat sich - wohl wissend, dass eine Strukturempfehlung immer auch unterschiedliche Einschätzungen hinsichtlich einer qualitativen Einstufung der Teilnehmer hervorrufen kann - ausführlich mit diesen Empfehlungen auseinandergesetzt. Es ist ihm klar, dass derartige Empfehlungen, die quasi als eine qualitative Einstufung von schmerztherapeutischen Einrichtungen aufgefasst werden können, zu Recht mit erheblicher kritischer Aufmerksamkeit bezüglich möglicher Auswirkungen wahrgenommen werden. Das Editorial soll dazu dienen, den Standpunkt des Präsidiums der DGSS zusätzlich zu verdeutlichen, um damit eine konstruktive Diskussion im Sinne eines nationalen Konsenses anzustoßen. ten in der Praxis sollte auf dem aktuellen Wissensstand der klinischen und experimentellen Forschung basieren, deren Umsetzung in die Praxis und Qualitätssicherung klar definierter Strukturen bedarf. Bisherige Konzepte in dieser Richtung basieren vor allem auf der Verabredung von Kriterien für die Abrechenbarkeit schmerztherapeutischer Leistungen im ambulanten (Qualitätssicherungsvereinbarung) und stationären Versorgungsbereich (Operationen- und Prozedurenschlüssel zur multimodalen Schmerztherapie). Trotz dieser Vereinbarungen bestehen sowohl eine sehr uneinheitliche und heterogene Versorgungsstruktur in der Schmerzmedizin mit erheblichen Unterschieden in der Ausstattung und Qualität der Versorgung als auch beeindruckende Unterschiede in der Vergütung. In einem ersten Schritt sind deshalb von der Ad-hoc-Kommission Multimodale Schmerztherapie im Auftrag der DGSS bereits Strukturempfehlungen vorgelegt worden [3], und es wird aufbauend auf dieser Arbeit aktuell an Kriterien der Prozess- und Ergebnisqualität gearbeitet. Die Ad-hoc-Kommission Strukturempfehlungen für schmerztherapeutische Einrichtungen hatte jetzt die Aufgabe, einen übergeordneten Entwurf für die Strukturierung sämtlicher schmerzmedizinischen Einrichtungen zu entwickeln.

\section{Wunsch und Wirklichkeit klaffen auseinander}

Bisherige Analysen der schmerztherapeutischen Versorgungssituation in Deutschland haben erste Ergebnisse zur Strukturqualität der stationären [4] und ambulanten Versorgung $[5,6]$ erbracht. Ein wesentliches Ergebnis der Befragung zur stationären Versorgung war, dass das in der Umfrage ermittelte Angebot schmerztherapeutischer Leistungen in Quantität und Qualität stark überschätzt wurde [6]. Plausibilitätsprüfungen ließen den Schluss zu, dass es sich bei vielen Angaben eher um Wunschdenken, bzw. den Wunsch der besonders positiven Selbstdarstellung, handelte als um realistische Einschätzungen, die die wirkliche Versorgungssituation widerspiegeln. Mit solchen Zahlen und Strukturen ist jedoch niemandem gedient. Im Gegenteil suggerieren sie eine qualitativ und quantitativ ausreichende Versorgung, die real gar nicht vorhanden ist.

In seiner Analyse zur schmerztherapeutischen Strukturqualität bei niedergelassenen Ärzten kam E. Lang bereits $2000 \mathrm{zu}$ dem Schluss, dass die über die Schmerztherapievereinbarung bzw. die Schmerzgesellschaften definierten Strukturqualitätsmerkmale und die für eine sinnvolle Therapie bei chronischen Schmerzen für notwendig gehaltene multimodale Prozessqualität oft nicht eingehalten wird [5]. Ein ähnliches Ergebnis zeigt die umfassende Analyse der ambulanten schmerztherapeutischen Versor- 
gungssituation von Kayser et al. [6], in der u. a. gezeigt werden konnte, dass sinnvolle und notwendige strukturqualitative Kriterien in unterschiedlichem Maße von vielen schmerztherapeutischen Einrichtungen nicht erfüllt wurden.

In allen drei Befragungen wurde insbesondere deutlich, dass interdisziplinäre und multiprofessionelle Strukturen in der aktuellen Versorgung nur in sehr eingeschränktem Ausmaß vorgehalten werden.

Die wissenschaftlichen Erkenntnisse der letzten Jahre haben aber deutlich gemacht, wie zentral ein solches Vorgehen in der Behandlung chronischer Schmerzen ist. Entsprechende Behandlungskonzepte sollten daher möglichst frühzeitig integriert werden. Auch bzgl. der multimodalen Behandlung gibt es ein Definitionsproblem, d. h. es gibt eine Fülle von entsprechenden Angeboten, die die Kriterien einer multimodalen Behandlung - wie die DGSS sie versteht [2] und wie sie z. B. in der Nationalen Versorgungsleitlinie Kreuzschmerz beschrieben sind - nicht erfüllen [7]. Die Ergebnisse des aktuellen Health Technology Assessment zur Lage der Schmerzmedizin im Auftrag des Bundesgesundheitsministeriums beschreiben diese erheblichen Defizite der schmerzmedizinischen Versorgung in Struktur und Qualität und verdeutlichen damit den Handlungsbedarf in dieser Richtung [8].

\section{- Im Qualitätsmanagement stellt die Struktur die Basis für die Prozess- und Ergebnisqualität dar.}

Eine zuverlässige Erfassung der Qualität ist nur bei vergleichbaren und akzeptierten Strukturmerkmalen möglich. Hier steht die Schmerzmedizin am Anfang der Entwicklung, die in anderen Bereichen wie z. B. den zertifizierten Zentren für die Behandlung von Krebserkrankungen der Deutschen Krebsgesellschaft e. V. [9] - bereits deutlich weiter fortgeschritten ist.

Ein unabdingbares Kriterium von Qualitätsmerkmalen ist dabei ihre (unabhängige) Überprüfbarkeit. Die notwendige interdisziplinäre und multiprofessionelle Kooperation in der Schmerztherapie muss kriterienbezogen nachprüfbar institutionalisiert sein und nicht ein hohles Papierbekenntnis. Erst auf dieser
Grundlage können in Zukunft dann Projekte zur Sicherung der Qualität sinnvoll und aussagekräftig anknüpfen. Dabei hat das Präsidium der DGSS die Hoffnung, dass mithilfe einer breiten Beteiligung schmerztherapeutischer Einrichtungen an einer Kerndokumentation der Nachweis von der Notwendigkeit und Effizienz schmerztherapeutischer Leistungen möglich wird. Die Definition strukturqualitativer Merkmale und eine Vergleichbarkeit verschiedener Stufen schmerztherapeutischer Versorgung ist dafür eine notwendige Voraussetzung.

\section{Das medizinisch Wirksame und ökonomisch Sinnvolle fordern}

Eine zentrale wissenschaftliche Zielsetzung sieht die DGSS im Bereich der Versorgungsforschung, auch hierfür schafft die Definition von Strukturqualität eine wesentliche Voraussetzung. Die DGSS wird die Versorgungsforschung in den kommenden Jahren zu einem zentralen Anliegen entwickeln, um die Krankenund Gesundheitsversorgung der Patienten mit akuten und chronischen Schmerzen weiter zu optimieren. Mit der Versorgungsforschung verbindet sich die Vorstellung, dass Wissenschaft helfen kann, durch ein besseres Verständnis der patientennahen Prozesse innerhalb des hochkomplexen Systems der medizinischen Versorgung innovative Entwicklungen unter dem Leitgedanken von Qualität und Kosteneffektivität möglichst zeitnah und breitenwirksam zu implementieren. Nach Meinung der DGSS wird die Bereitstellung von Daten und Fakten am ehesten geeignet sein, mit politischem Gewicht bei Politikern und Versorgern die Verbesserung der nationalen Versorgungsstruktur einzufordern. Die Entwicklung und Implementierung von KEDOQS (Kerndokumentation und Qualitätssicherung) in der Schmerztherapie und die hier diskutierten Strukturempfehlungen für schmerztherapeutische Einrichtungen sind wichtige Signale, um fundierte wissenschaftliche Daten als Grundlage für neue Konzepte der Versorgung zu generieren.

Aber auch für die Verhandlungen um ausreichende Strukturen und angemessene Vergütung schmerztherapeutischer Leistungen mit Politikern und Kostenträ- gern ist eine transparente und nachprüfbare Strukturqualität notwendig, welche die zur Versorgung der Schmerzpatienten notwendigen, besonderen Qualitätsmerkmale darstellt. Diese Strukturempfehlung soll Schmerztherapeuten bei Gründungen von Praxisgemeinschaften, Medizinischen Versorgungszentren und Klinikabteilungen in die Lage versetzen, gegenüber den Kassenärztlichen Vereinigungen, Kostenträgern und Klinikverwaltungen mit breit konsentierter und konkreter Rückendeckung zu verhandeln.

Wenn wir die unzureichende Versorgung unserer Schmerzpatienten beklagen, insbesondere die fehlende Anerkennung und Honorierung interdisziplinärer multimodaler Programme, dann müssen wir auch die erforderlichen Strukturen benennen, auch und gerade wenn sie derzeit nur schwer realisiert werden können. Wir, d. h. alle Schmerzgesellschaften, sollten Einigkeit hinsichtlich der Forderung nach einer qualitativ hochwertigen schmerztherapeutischen Versorgung demonstrieren, die weniger von Partikularinteressen, als von einer ernst $\mathrm{zu}$ nehmenden und überzeugenden Gesamtkonzeption medizinisch wirksamer und ökonomisch sinnvoller Strukturen bestimmt wird, um mit Politikern und Geldgebern im Gesundheitssystem in einen konstruktiven Dialog einzusteigen zu können.

Die explizite inhaltliche Darstellung der Empfehlungen bleibt der Publikation in diesem Heft vorbehalten. Nur so viel: Die hier vorgestellte Definition sieht eine 4-fache Abstufung schmerztherapeutischer Einrichtungen vor. Dabei hat die Kommission auch bereits einige Prozesskriterien, wie z. B. Anzahl von Patienten und Anforderungen an die Dokumentation, mit einfließen lassen. Ganz wichtig ist es uns, klarzustellen, dass eine Struktur der Stufe 4 die Basis einer qualitativ hochwertigen und zeitgemäßen Versorgung darstellt und keine qualitative $\mathrm{Ab}$ wertung impliziert.

\section{Wie geht es weiter?}

Die Empfehlungen der Ad-hoc-Kommission reflektieren aus unserer Sicht diejenigen Kriterien, die eine Voraussetzung dafür darstellen, Schmerzpatienten angemessen und qualitativ hochwertig $\mathrm{zu}$ 
behandeln. Die DGSS kann als zentrale wissenschaftliche Fachgesellschaft in der Schmerzmedizin zwar Strukturen schmerztherapeutischer Einrichtungen definieren, für eine tragfähige Umsetzung in die Praxis ist jedoch eine breitere Akzeptanz notwendig und sinnvoll. Daher suchen wir die Diskussion, Abstimmung und letztendlich den Konsens mit anderen Fachgesellschaften und Berufsverbänden, um gerade im gesundheitspolitischen Rahmen die Voraussetzung der Finanzierbarkeit der geforderten Strukturen einzufordern und zu erreichen. Nach unserer Auffassung sind die jetzt vorliegenden Empfehlungen ein wichtiges Signal, um diese Diskussion um die zukünftige Gestaltung und Entwicklung der Schmerzmedizin in Deutschland anzustoßen. Die DGSS wird diesen Prozess in den kommenden Monaten aktiv moderieren. Wir werden das Gespräch mit den Gesellschaften und Verbänden suchen, aber auch Rückmeldungen und Statements von Einzelpersonen sind hochwillkommen. $\mathrm{Zu}$ diesem konstruktiven Dialog möchten wir Sie hiermit herzlich einladen!

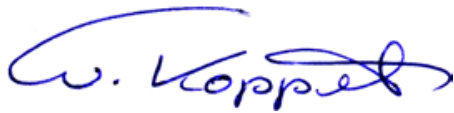

W. Koppert

für die Mitautoren

\section{Korrespondenzadresse}

\section{Prof. Dr. W. Koppert}

Klinik für Anästhesiologie u. Intensivmedizin,

Medizinische Hochschule Hannover

Carl-Neuberg-Str. 1, 30625 Hannover

Koppert.Wolfgang@mh-hannover.de

\section{Literatur}

1. Loeser J (1991) Desirable characteristics for pain treatment facilities. In: Bomb MR, Charlton JE, Woolf $\mathrm{CJ}$ (eds) Proceeding of the $\mathrm{VI}^{\text {th }}$ World Congress on pain. Elsevier, Amsterdam, S 411-415

2. Sabatowski R, Maier C, Willweber-Strumpf A et al (2011) Empfehlungen zur Klassifikation schmerztherapeutischer Einrichtungen in Deutschland. Schmerz 25

3. Arnold B, Brinkschmidt T, Casser HR et al (2009) Multimodale Schmerztherapie - Konzepte und Indikation. Schmerz 23: 112-120

4. Lindena G, Hildebrandt J, Diener HC et al (2004) Schmerztherapeutische Angebote an Kliniken in Deutschland. Schmerz 18: 10-16

5. Lang E (2000) Schmerztherapeutische Strukturqualität bei niedergelassenen Ärzten. Gibt es einen Standard? Schmerz 14: 226-230
6. Kayser H, Thoma R, Mertens E et al (2008) Struktur der ambulanten Schmerztherapie in Deutschland. Schmerz 22: 424-432

7. Bundesärztekammer (BÄK), Kassenärztliche Bundesvereinigung (KBV), Arbeitsgemeinschaft der Wissenschaftlichen Medizinischen Fachgesellschaften (AWMF) (2010) Nationale Versorgungsleitlinie Kreuzschmerz - Langfassung. Version 1.0 (letzter Zugriff: 30.11.2010). URL: http://www.versorgungsleitlinien.de/themen/kreuzschmerz

8. Dietl M, Korczak D (2011) Versorgungssituation der Schmerztherapie in Deutschland. Schriftenreihe des Health Technology Assessment in Deutschland, Bd. 111. DIMDI, Köln

9. Deutsche Krebsgesellschaft e. V., Zertifizierte Zentren für die Behandlung von Krebserkrankungen. URL: http://www.krebsgesellschaft.de/wub_zertifizierte_zentren_uebersicht.html

\section{Erwartungshaltung bestimmt Wirkung von Schmerzmedikamenten}

Eine Schmerztherapie wirkt besser, wenn Patienten sich viel von der Behandlung versprechen. Zu dieser Erkenntnis gelangt eine Studie, bei der Probanden einem Hitzereiz ausgesetzt wurden. Während des Versuchs erhielten die Testpersonen mittels Infusion ein stark wirksames, opioidhaltiges Schmerzmittel (Remifentanil). In der ersten Gruppe bekamen die Probanden das Schmerzmittel in einer "verdeckten" Infusion und rechneten nicht mit einer Schmerzlinderung. Nach Verabreichung des Medikaments nahm die ursprüngliche Schmerzintensität ab. In der zweiten Gruppe erhielten die Probanden das Schmerzmittel, nachdem Ihnen die Verabreichung angekündigt wurde. In dieser Gruppe verdoppelte sich der schmerzlindernde Effekt bei identischer Medikamentendosierung. Gänzlich aufgehoben wurde der schmerzlindernde Effekt, wenn den Probanden gesagt wurde, dass sie keine Therapie mehr erhielten und es gleich stärker schmerzen könnte. Obwohl ihnen ohne ihr Wissen weiter das Analgetikum verabreicht wurde, stieg die Schmerzintensität wieder auf den Ausgangswert an. Die Wissenschaftler postulieren, dass eine negative Erwartung den Effekt eines Schmerzmedikaments zerstören kann. Die Ergebnisse könnten für die Behandlung von Patienten mit chronischen Schmerzen bedeutsam sein. Nach Meinung der Wissenschaftler könnte es bereits helfen, Patienten gezielter über ihre Behandlung aufzuklären, um positive Erwartungen zu wecken und negative zu vermeiden.

Literatur: Bingel U, Wanigasekera V, Wiech Ket al (2011) The impact of treatment expectation on drug efficacy: Imaging the Analgesic Benefit of the Opioid Reminfentanil. Sci Transl Med 3(70):70ra14

Quelle: Universitätsklinikum Hamburg-Eppendorf, www.uke.de 